\title{
Diseño de una propuesta didáctica para el uso de simuladores virtuales en la rama sanitaria de Formación Profesional
}

\section{Design of a didactic proposal for the use of virtual simulators in the health area of Vocational Training}

\author{
Daniel Romero López \\ Universidad de las Islas Baleares \\ romerolopezdani@gmail.com \\ Bárbara de Benito Crosetti \\ Universidad de las Islas Baleares \\ barbara.debenito@uib.es
}

Recibido: $11 / 06 / 2019$

Aceptado: $18 / 12 / 2019$

Publicado: 01/06/2020

\section{RESUMEN}

En este artículo se presenta el diseño, desarrollo y validación de una propuesta didáctica basada en el uso de simuladores virtuales, con la que se pretende conseguir una mejora en la preparación práctica del alumnado, llevada a cabo en un ciclo de Formación Profesional (FP) del ámbito sanitario. Las posibilidades de trabajar experiencias prácticas son escasas durante la formación del alumnado en los centros de Formación Profesional, debido a la carencia de espacios y oportunidades, algo fundamental para la preparación del periodo final de prácticas en los centros sanitarios. Para ello se utiliza un simulador virtual en el módulo de Tomografía Computarizada, permitiendo poner en práctica los conocimientos teóricos que van adquiriendo los alumnos durante su formación. Los resultados obtenidos permiten apreciar las ventajas de la simulación como medio de aprendizaje en la enseñanza relacionada con el ámbito clínico.

\section{PALABRAS CLAVE}

Simuladores virtuales; Formación Profesional; formación práctica; recurso digital.

\section{ABSTRACT}

This article presents the design, development and validation of a didactic proposal based on the use of virtual simulators, with the intention of achieving an improvement in the practical preparation of students, carried out in a cycle of Vocational Training in the health field. The possibilities of working with practical experiences are scarce during the training of students in vocational training centers, due to the lack of spaces and opportunities, which is fundamental for the preparation of the final period of internships in health centers. For this reason, a virtual simulator is used in the Computerized Tomography module, allowing to put into practice the theoretical knowledge that students acquire during their training. The results obtained allow us to appreciate the advantages of simulation as a learning tool in teaching related to the clinical field.

\section{KEYWORDS}

Virtual simulators; Vocational Training; practical training; digital resources. 


\section{CITA RECOMENDADA}

Romero, D. \& De Benito, B. (2020). Diseño de una propuesta didáctica para el uso de simuladores virtuales en la rama sanitaria de Formación Profesional. Revista Interuniversitaria de Investigación en Tecnología Educativa, 8, 1-16. http://dx.doi.org/10.6018/riite.383431

\section{Principales aportaciones del artículo y futuras líneas de investigación:}

- Conocer el nivel de aceptación del uso de simuladores virtuales en Formación Profesional.

- Integrar los simuladores virtuales en Formación Profesional.

- Contribuir a la formación práctica del alumnado de Formación Profesional del ámbito sanitario.

\section{INTRODUCCIÓN}

El desarrollo y acceso de las Tecnologías de la Información y la Comunicación (TIC) ha impulsado su uso prácticamente en todos los niveles o etapas del ámbito educativo. Por lo tanto, la Formación Profesional (FP) presencial también podría beneficiarse del importante papel del avance tecnológico, al obtener herramientas que permiten al alumnado la adquisición de conocimiento y habilidades, así como la consecución de un aprendizaje más activo y eficaz.

Sin embargo, el uso que se hace de estos recursos en las modalidades presenciales de FP para favorecer el aprendizaje del alumnado es escaso y, en la mayoría de centros, se sigue apostando por una metodología tradicional basada en explicaciones magistrales, siendo la integración de las TIC en el currículo todavía una tarea compleja y desafiante (González y de Pablos, 2015). Además, tal y como indican Silva y Maturana (2017), las estrategias tradicionales no permiten dar respuesta a las necesidades formativas de los actuales estudiantes en la Educación Superior.

En este sentido, las metodologías activas de aprendizaje, como la simulación, surgen como las herramientas más adecuadas de un entorno educativo que prepare al alumnado para los retos que la sociedad actual enfrenta (Rodríguez, Maya y Jaén, 2012).

Además, una de las desventajas a las que se enfrentan los estudiantes durante su formación es la falta de espacios y oportunidades donde poner en práctica sus conocimientos (Cárdenas, Sánchez y Castillo, 2016). Frente a esto, los simuladores virtuales permiten, en primera instancia, un cambio de ambiente de enseñanza-aprendizaje representado por la modelación de situaciones reales, facilitando el logro de determinados objetivos educativos; el uso de estos recursos digitales también facilita la realización de ejercicios prácticos por parte de los estudiantes en situaciones controladas.

De acorde a esto, en el presente artículo se analiza el diseño e implementación de una estrategia didáctica basada en el uso de simuladores virtuales; a través de la aplicación de estos recursos digitales, con los que se pretende favorecer el logro de determinados beneficios en el alumnado, como conseguir una mejor asimilación de contenidos teóricos y preparación para realizar las prácticas externas en los centros sanitarios.

\section{ESTADO DEL ARTE}

\subsection{El uso potencial de las TIC en Formación Profesional}

Matia (2016) defiende que uno de los objetivos de la FP en nuestro país es posibilitar el tránsito entre el sistema formativo y el mundo laboral, siendo este nivel formativo el que presenta actualmente los estudios profesionales que más se acercan a la realidad del mercado laboral. Según Pin y García (2018), la empleabilidad desempeña un papel esencial en la capacidad de la FP para atraer a los jóvenes, lo que ha provocado que el crecimiento de la misma haya sido progresivo y constante durante los últimos años en España. Los estudios de 
FP han pasado de estar en un segundo plano a ser reconocidos como los que otorgan una mejor cualificación, siendo uno de los puntos fuertes las prácticas que realizan los alumnos en las empresas privadas o instituciones públicas.

Para Alcides (2008), en las Instituciones de Educación Superior, donde se incluyen las modalidades de FP, la incorporación de las TIC facilita el desarrollo de habilidades y competencias para el desenvolvimiento social y cultural, fortalece la creatividad, la lógica y el razonamiento, gracias a los medios audiovisuales y digitales. Sin embargo, muchos de los centros que imparten estas etapas todavía siguen apostando por la metodología tradicional, exclusivamente presencial y sin apoyarse en este uso de herramientas digitales o entornos de formación.

Según diversos autores (García, Reyes y Godínez, 2018; Gibelli, 2014; Silva y Maturana, 2017), para que los estudiantes puedan adquirir el conocimiento y las habilidades esenciales para desenvolverse adecuadamente en el ámbito social y profesional del siglo XXI, es necesario modificar la forma de concebir el proceso de enseñanza - aprendizaje y centrarlo en el alumno, ya que las estrategias tradicionales que se han utilizado hasta ahora no permiten dar respuesta a las necesidades formativas de los actuales estudiantes de FP. El docente debe de cambiar de un enfoque didáctico, basado en la mera transmisión de información, a otro que promueva la participación de los estudiantes y su aproximación al ámbito profesional, algo en lo que pueden ser muy útiles las herramientas digitales. Así mismo, de acuerdo con Salinas (2004), para adaptarse a las necesidades de la sociedad actual, las instituciones de Educación Superior deben flexibilizarse y desarrollar vías de integración de las TIC en los procesos formativos.

La modalidad presencial en Educación Superior no ha sido ajena a los cambios que en la sociedad del conocimiento han aportado las TIC (Alcides, 2008). Las TIC, en los procesos formativos, han creado cambios en la forma de organización, interacción entre los sujetos y, especialmente, en el modo en que se aprende y se construye el conocimiento. Su utilización, como metodología integradora en el proceso de enseñanza-aprendizaje, ofrece nuevos métodos de trabajo en el aula y contribuye a la creación de nuevos ambientes de aprendizaje mediante los que los estudiantes acceden al conocimiento de una forma distinta.

En su investigación con el uso de las TIC, Coscollola y Fuentes (2010) también llegaron a la conclusión de que, entre algunas de las ventajas de estas herramientas en los procesos de enseñanza-aprendizaje, las TIC impiden reducir al alumno a un sujeto pasivo, ya que permiten la interacción en tiempo real, el intercambio de experiencias y la aproximación a realidades lejanas a la metodología tradicional.

\subsection{Simuladores virtuales como estrategia formativa}

Un simulador virtual es un programa digital que trata de representar posibles situaciones de la vida real, poniendo a disposición del usuario las funcionalidades de un producto o técnica para probarlo por sí mismo. Esto permite al estudiante cambiar algunos parámetros o variables de su entorno, ejecutar el modelo y analizar los resultados, asemejándose a lo que ocurre en un entorno real (Contreras, García y Ramírez, 2010). Una de las desventajas a las que se enfrentan los estudiantes durante su formación, es la falta de espacios y oportunidades donde poner en práctica sus conocimientos. En el ámbito educativo, la utilización de simuladores virtuales se está extendiendo, caracterizándose como una de las tecnologías emergentes con aplicaciones en diferentes áreas: ciencias de la salud, economía, matemáticas, física etc.

Para la enseñanza de habilidades clínicas, ámbito de estudio donde se centra el presente artículo, es necesario combinar la teoría, con la formación en entornos clínicos reales; sin embargo, debido a distintas restricciones de tiempo, espacio y costes, no se produce este acceso durante la formación del alumnado (Cárdenas, Sánchez y Castillo, 2016). Para que los estudiantes puedan adquirir los conocimientos y habilidades esenciales que les permitan desenvolverse adecuadamente en los centros de prácticas, es necesario realizar una enseñanza centrada en la adquisición de estas competencias y no únicamente la de conocimientos teóricos. 
En este sentido, diversos autores (Cárdenas, Sánchez y Castillo, 2016; Corvetto et al., 2013) exponen que el crecimiento de los simuladores virtuales en el ámbito clínico ha sido muy significativo, estos últimos avances han puesto a disposición de los estudiantes en formación tecnologías que permiten la reproducción de eventos clínicos con suficiente fidelidad para permitir su participación de una forma realista y significativa.

Los simuladores virtuales se presentan como una de las estrategias para capacitar a los estudiantes y reforzar los conceptos teóricos, permitiendo al usuario interactuar y familiarizarse con ambientes a los que se pueden llegar a enfrentar en el futuro, así como aprender a manejar posibles situaciones y la forma de reaccionar ante éstas. Es así, que el estudiante puede practicar las ocasiones necesarias en escenarios virtuales, contando además, con la libertad de cometer errores, aprender de ellos y así alcanzar el dominio de la competencia clínica (Cárdenas, Sánchez y Castillo, 2016).

Tal y como han demostrado diversos estudios (Franco y Álvarez, 2007), el aprendizaje a través de simuladores es un método de los más eficaces para adquirir habilidades y destrezas, mejorando la tasa media de retención en el aprendizaje. Gracias a este tipo de herramientas es posible que los alumnos recreen las condiciones y situaciones que se dan en el medio laboral del futuro profesional. Se crea así una nueva vía para que los alumnos puedan experimentar y desarrollar competencias de su ámbito profesional en escenarios donde no siempre tienen acceso.

\section{MÉTODO}

La metodología de investigación de este estudio ha sido la Investigación Basada en Diseño (IBD), por ser un tipo de investigación orientada a la innovación educativa y cuya característica fundamental es la introducción de un elemento transformador de una situación educativa (De Benito y Salinas, 2016). Esta metodología tiene como meta principal proponer soluciones a problemas detectados en la realidad educativa a través de programas, materiales o estrategias didácticas innovadoras en el ámbito estudiado.

En una realidad como la mencionada anteriormente en Formación Profesional, donde es habitual que los alumnos no estén preparados para el periodo de prácticas, debido a la falta de espacios donde poder aplicar los conocimientos adquiridos, se hace necesario plantear una metodología que permita adaptar la enseñanza a estas condiciones particulares.

En este contexto, la investigación se trata de una propuesta didáctica que se implementó en el curso 2018-2019 en un centro de Formación Profesional de la Región de Murcia, con estudiantes de segundo curso del ciclo de Imagen para el Diagnóstico y Medicina Nuclear; cuya finalidad era mejorar la preparación práctica del alumnado de Formación Profesional a través del uso de las TIC.

\subsection{Objetivos}

En este proyecto, la situación donde se pretende introducir un elemento transformador es la preparación con la que llegan a las prácticas externas los alumnos, para que adquieran experiencia en la parte práctica del ámbito clínico, ya que lo que más se trabaja en estas modalidades durante el curso es la adquisición de conocimientos teóricos.

Por ello, el objetivo general es diseñar y validar una propuesta didáctica basada en el uso de simuladores virtuales, con la que se pretende conseguir una mejora en la preparación práctica del alumnado antes de comenzar el periodo de trabajo en los centros asignados.

En cuanto a los objetivos específicos que se pretenden lograr:

1. Justificar la idoneidad de la herramienta utilizada.

2. Analizar el grado de aceptación de estos recursos por parte del alumnado.

3. Determinar si esta estrategia, basada en el uso de simuladores virtuales, favorece la aplicación de los conceptos teóricos en situaciones prácticas. 


\subsection{Participantes}

El grupo de estudio está formado por un total de 48 alumnos de segundo curso de este ciclo de Formación Profesional Superior, dividido en dos grupos, uno de 30 alumnos y el otro de 18. Respecto a la edad de los participantes, el promedio es de unos 22 años, siendo en su mayoría jóvenes de entre 18 y 25 años.

La propuesta se llevó a cabo en el módulo de Tomografía Computarizada (TC) perteneciente al 2. ํ curso del ciclo de Imagen para el Diagnóstico y Medicina Nuclear, en el curso 2018-2019.

El equipo investigador estaba conformado principalmente por el profesor de la materia (el investigador); también fueron informados del recurso que se deseaba incorporar en el programa formativo el ayudante técnico de imagen y diagnóstico, y el coordinador del ciclo.

\subsection{Diseño de la propuesta}

\subsubsection{Simulador utilizado}

El Ministerio de Educación (MEC), a través del Instituto Nacional de Tecnologías Educativas y de Formación del Profesorado (INTEF), ha desarrollado numerosos recursos educativos digitales, entre los que se encuentran una serie de simuladores virtuales, que se adaptan al currículo de distintos ciclos de Formación Profesional.

Con el uso de este simulador virtual en el módulo de Tomografía Computarizada se pretendía que los estudiantes experimentaran el trabajo de un técnico de imagen, a modo de reafirmación de los contenidos teóricos trabajados en clase y, como se comenta en apartados anteriores, con el objetivo de simular una situación práctica que prepare al alumnado para la FCT (Formación en Centros de Trabajo).

Se instalaron en los ordenadores del centro los simuladores virtuales específicos de esta asignatura (Tomografía Computarizada), donde el usuario trabaja en los ambientes característicos de un técnico de imagen y diagnóstico: sala del tomógrafo, cabina del software y sala de espera.

Estos simuladores virtuales inician con una breve introducción teórica sobre los componentes y características de cada tipo de protocolo, esto, antes de dar comienzo a la práctica simulada. La simulación se lleva a cabo en primera persona, donde el usuario actúa como un técnico de imagen que debe realizar un volante médico específico: comprobación del equipo necesario para llevar a cabo la exploración, posicionamiento del paciente, ajuste de parámetros del equipo y control de calidad de las imágenes obtenidas (figura 1).

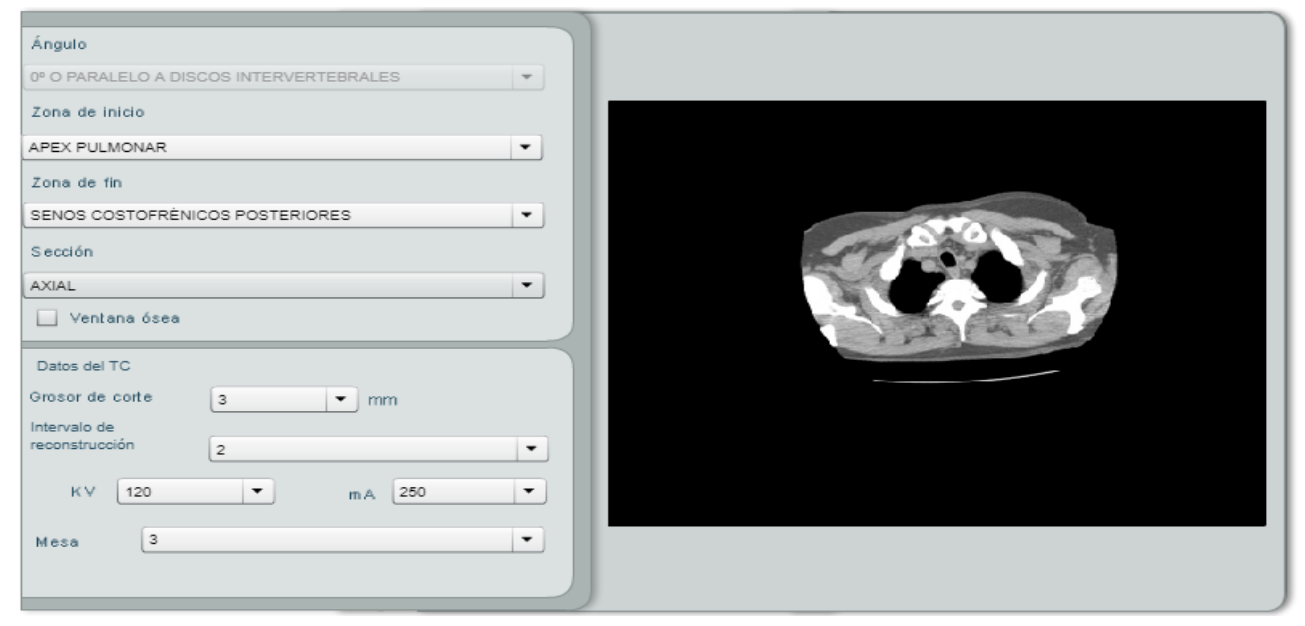

Figura 1 . Obtención de imágenes a través del simulador de tomografía

Finalmente, una vez terminado el protocolo, el simulador genera un informe donde se puede observar la puntuación del usuario y los fallos que ha cometido durante el proceso; 
planteando la posibilidad de utilizar estos informes como una forma de evaluar los conocimientos prácticos de la asignatura.

\subsubsection{Propuesta}

Una vez trabajados en clase los conceptos relacionados con un área anatómica concreta (posicionamiento del paciente, posibles patologías asociadas, parámetros necesarios, etc.), se realizó la experiencia simulada en el aula de informática, con el fin de que los alumnos pusieran en "práctica" estos conocimientos adquiridos.

Para ello, de las 8 horas semanales de este módulo, 6 se dedicaron a las explicaciones teóricas y otras actividades en el aula, y 2 al trabajo práctico en el simulador y puesta en común de resultados. Destacar el hecho de que aquí se presenta un gran modelo para trabajar en la formación presencial, debido a que durante la realización de la simulación el alumno recibe apoyo y motivación en tiempo real del docente.

\subsection{Técnicas e instrumentos}

Según De Benito y Salinas (2016), la IBD no dispone de una metodología propia, apoyándose en cualquier tipo de metodología que mejor se adapta a solucionar el problema definido, aunque en este caso su naturaleza es principalmente cuantitativa, debido a los instrumentos utilizados para la obtención de los resultados. En la presente investigación se utilizaron las siguientes técnicas e instrumentos:

\subsubsection{Evaluación del simulador}

El simulador utilizado se evaluó previamente por el docente y el técnico de imagen, con el fin de analizar la validez del instrumento para el objetivo de aprendizaje propuesto. Tras poner en práctica el simulador en clase, los alumnos también evaluaron la herramienta a través de un instrumento donde se analizan distintas dimensiones del simulador.

Las dimensiones que conformaron el instrumento y los ítems, se han obtenido de la investigación llevada a cabo por Cabero y Costas (2016) sobre la utilización de simuladores. Cada ítem se evaluó con unos valores de 1 a 4, representando el valor 1 la característica "En desacuerdo" y el valor 4 "Totalmente de acuerdo".

\subsubsection{Cuestionarios}

En la investigación se utilizó un cuestionario previo para conocer el uso de recursos tecnológicos en el centro, así como el conocimiento que presenta el alumnado en relación a los recursos digitales y la opinión sobre su preparación práctica. Para ello, se planteó un cuestionario cerrado de respuesta múltiple, pero donde también podían añadir información adicional a la respuesta señalada.

Debido a que se trata de una investigación centrada en un área muy concreta, no se ha encontrado ningún cuestionario que se adapte completamente a las necesidades del estudio. Por esa razón, se elaboró un cuestionario propio centrado en las dimensiones que se pretendían estudiar en la investigación (uso de las TIC en el aula y preparación práctica). Los datos se recogieron a finales del primer trimestre del curso, previo a la aplicación de esta propuesta.

También se realizó un cuestionario final tras la utilización de los simuladores virtuales, para conocer la opinión sobre el recurso utilizado y si había cambiado su percepción sobre la preparación para las prácticas externas. En este caso también se trata de un cuestionario cerrado de respuesta múltiple que pretendía recoger información de dimensiones relacionadas con los simuladores utilizados y la propuesta metodológica utilizada en el segundo trimestre.

\subsubsection{Rendimiento académico}

Se ha visto conveniente añadir en este estudio, información sobre el rendimiento académico de los alumnos, con el objetivo de evidenciar que el uso de estos simuladores puede haber contribuido a una mayor comprensión o asimilación de conceptos. Para ello, se realizó una comparación de las calificaciones obtenidas en la $1 . \stackrel{a}{a}$ evaluación, donde las 
sesiones se basaban exclusivamente en explicaciones teóricas, y de la 2. a evaluación, donde se incorporaron los simuladores virtuales.

\subsubsection{Entrevista}

Así mismo, una vez empezado el periodo de prácticas externas en los centros clínicos, se recogió información del tutor de prácticas a través de una entrevista semiestructurada, con el objetivo de conocer la preparación del alumnado con la que habían llegado a las clínicas. La entrevista estaba enfocada a conocer el desempeño de los estudiantes en los centros de prácticas, centrándose en la técnica estudiada en la asignatura de Tomografía Computarizada, donde se desarrolla la metodología propuesta.

\section{RESULTADOS}

\subsection{Resultado del cuestionario previo}

Los resultados del cuestionario previo mostraron claramente la carencia del uso de recursos tecnológicos como apoyo en el aula durante la 1. a evaluación (figura 2). Aquí se aprecia que el $80 \%$ del alumnado encuestado considera que las TIC como apoyo en el aula han sido escasamente utilizadas durante su formación; mientras que el $20 \%$ restante que ha respondido a la pregunta con "mucho" y "a menudo" han acompañado sus respuestas con el uso de presentaciones e imágenes proyectadas en la pizarra digital para acompañar las explicaciones teóricas.

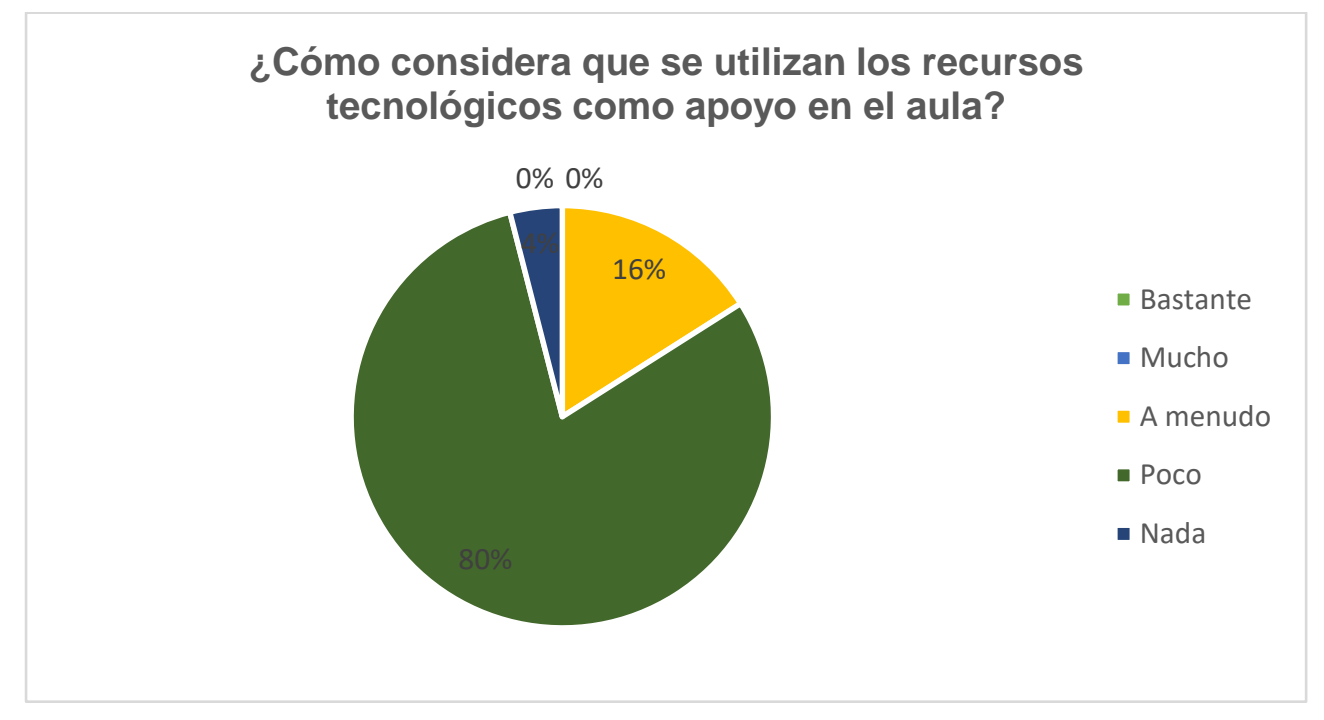

Figura 2. Uso de los recursos tecnológicos en el aula

También se pudo observar que la mayoría del alumnado no se consideraba realmente preparado para realizar las prácticas externas (figura 3), solo 11 de los 48 alumnos se consideraban "Bien" o "Bastante" preparados para la realización de esta etapa en las clínicas sanitarias. 


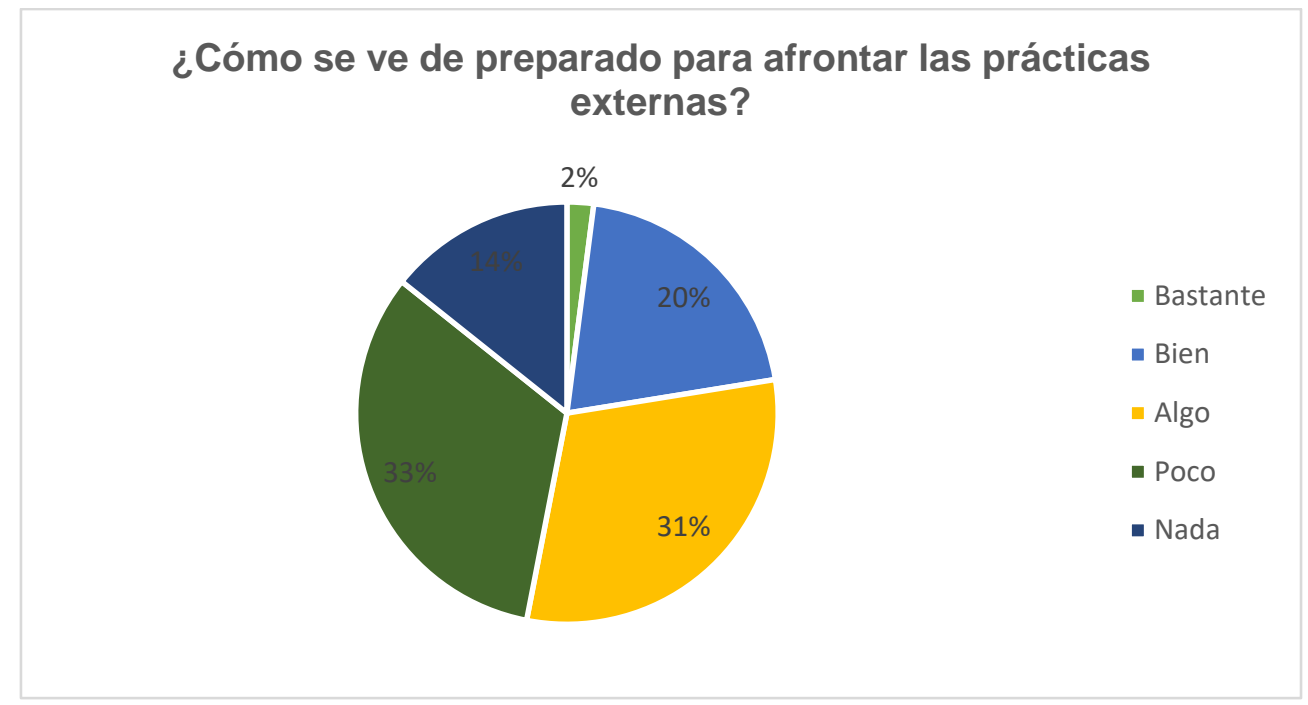

Figura 3. Preparación inicial para afrontar las prácticas externas.

Estas prácticas son de las más importantes del ciclo, y en el centro, año tras año, se aprecia la preocupación del profesorado y la jefatura de estudios porque el alumnado se muestre capaz de desenvolverse en los centros de prácticas. Algunos de los alumnos que escribieron en el espacio facilitado del cuestionario expusieron que esto se debe a que no han tenido contacto con experiencias prácticas a lo largo del ciclo.

\subsection{Resultado de la evaluación del simulador}

Para la evaluación del simulador, se llevó a cabo una valoración por el docente del módulo y el técnico de imagen, y otra por los usuarios potenciales, en este caso el alumnado (tabla 1). El objetivo de evaluar la herramienta no fue otro que considerar si se adaptaba a la realidad clínica, dentro de los límites que presentan este tipo de recursos.

Las evaluaciones del cuestionario por el docente y el técnico de imagen se realizaron antes de utilizar los simuladores con los alumnos, mientras que estos realizaron la evaluación tras tener la primera experiencia con la herramienta.

\begin{tabular}{|c|c|c|c|c|}
\hline Dimensión & Ítem & $\begin{array}{l}\text { Valoración } \\
\text { docente }\end{array}$ & $\begin{array}{l}\text { Valoración del } \\
\text { técnico }\end{array}$ & $\begin{array}{c}\text { Valoración } \\
\text { media } \\
\text { alumnos }\end{array}$ \\
\hline \multirow[t]{4}{*}{ Aspectos técnicos } & $\begin{array}{l}\text { Tamaño de gráficos y } \\
\text { letras }\end{array}$ & 2 & 3 & 3,12 \\
\hline & Velocidad de carga & 3 & 3 & 2,97 \\
\hline & Respuesta a acciones & 3 & 4 & 3,42 \\
\hline & Menús de ayuda & 4 & 4 & 3,10 \\
\hline \multirow[t]{4}{*}{ Contenidos } & Información actualizada & 3 & 2 & 2,75 \\
\hline & $\begin{array}{l}\text { Veracidad de los } \\
\text { contenidos }\end{array}$ & 4 & 4 & 3,21 \\
\hline & $\begin{array}{l}\text { Secuencia del } \\
\text { procedimiento }\end{array}$ & 4 & 4 & 3,84 \\
\hline & $\begin{array}{l}\text { Calidad de los } \\
\text { contenidos }\end{array}$ & 3 & 3 & 3,15 \\
\hline \multirow{2}{*}{$\begin{array}{l}\text { Organización de } \\
\text { la información }\end{array}$} & Incluye tutoriales & 3 & 2 & 2,83 \\
\hline & $\begin{array}{l}\text { Permite realizar } \\
\text { estudios específicos }\end{array}$ & 3 & 4 & 3,55 \\
\hline
\end{tabular}




\begin{tabular}{|c|c|c|c|c|}
\hline \multirow[t]{4}{*}{ Valor educativo } & $\begin{array}{l}\text { Ofrece } \\
\text { retroalimentación }\end{array}$ & 4 & 3 & 2,93 \\
\hline & Se adapta al módulo & 4 & 4 & 3,67 \\
\hline & $\begin{array}{l}\text { Los problemas } \\
\text { presentados se } \\
\text { adecúan } \\
\text { con la realidad } \\
\text { profesional * }\end{array}$ & 3 & 4 & - \\
\hline & $\begin{array}{l}\text { Las decisiones influyen } \\
\text { en el desarrollo del } \\
\text { protocolo }\end{array}$ & 2 & 3 & 2,43 \\
\hline \multirow[t]{4}{*}{ Diseño } & $\begin{array}{l}\text { Coherente con la } \\
\text { realidad clínica * }\end{array}$ & 3 & 3 & - \\
\hline & $\begin{array}{l}\text { Animaciones } \\
\text { adecuadas }\end{array}$ & 3 & 3 & 3,13 \\
\hline & Colores adecuados & 3 & 4 & 3,72 \\
\hline & $\begin{array}{l}\text { Distribución correcta } \\
\text { del equipo médico }\end{array}$ & 4 & 4 & 3,44 \\
\hline \multirow[t]{3}{*}{ Motivación } & Despierta interés & 4 & 3 & 3,25 \\
\hline & $\begin{array}{l}\text { Tiempo por protocolo } \\
\text { adecuado }\end{array}$ & 2 & 1 & 1,93 \\
\hline & Grado de atracción & 3 & 3 & 3,38 \\
\hline
\end{tabular}

Tabla 1. Resultados de la evaluación del simulador.

De forma general, como se puede observar en todos los ítems, salvo en uno: "Tiempo por protocolo adecuado" $(1,93)$, se muestran puntuaciones superiores a 2 , lo cual determina que el simulador fue positivamente valorado por los participantes.

En el análisis realizado por el docente y el técnico de imagen se observó que hay algunos ítems que también podrían implementarse, por ejemplo: "Tamaño de gráficos y letras", "Información actualizada", "Incluye tutoriales" o "Las decisiones influyen en el desarrollo del protocolo". En 5 ítems los valores alcanzan el valor de 3,5 o superior, incluido el ítem de "Coherente con la realidad clínica", y los referentes a que si el simulador se adapta a los contenidos tratados en el módulo y si sigue la secuencia estudiada de actuación.

\subsection{Resultado del cuestionario final}

En la figura 4 se puede comprobar la opinión de los alumnos respecto al uso de los simuladores virtuales aplicados al ámbito de estudio. En general, los participantes han encontrado interesante su utilización, siendo un $58 \%$ los que lo han calificado como muy útil por permitir poner en práctica los conocimientos teóricos adquiridos durante las clases magistrales. 


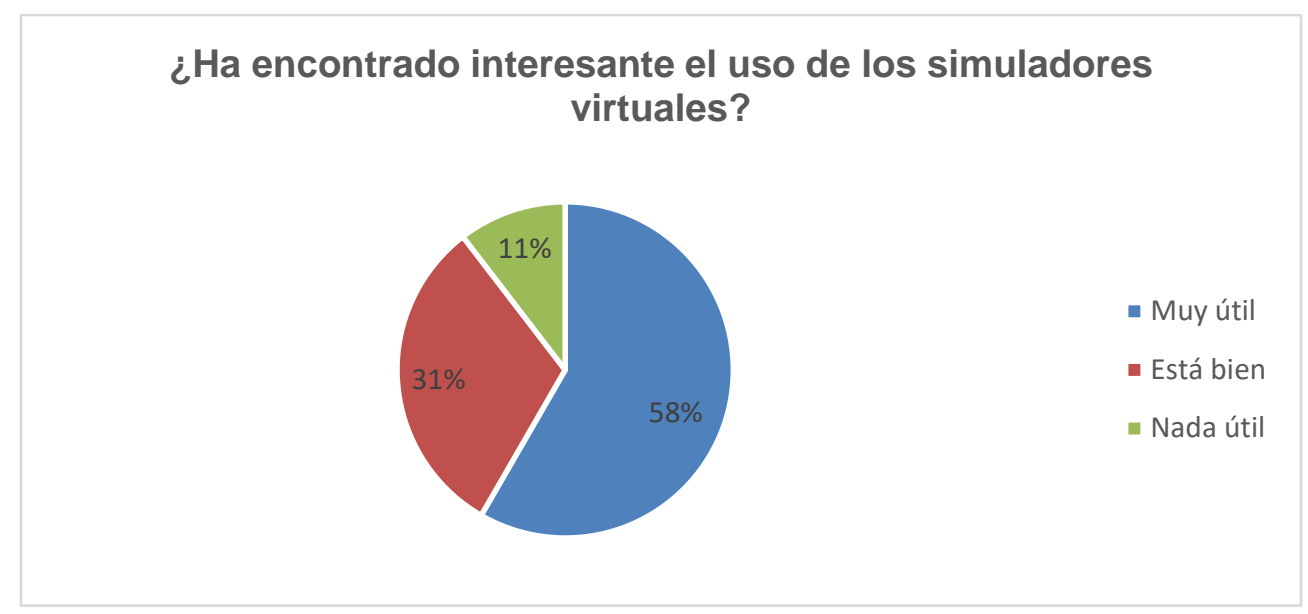

Figura 4. Interés en el uso de la herramienta.

En la figura 5 se puede observar cómo el uso de los recursos planteados en esta propuesta ha sido aceptado ampliamente por los estudiantes, como una mejora en su preparación respecto a la metodología empleada en la primera evaluación.

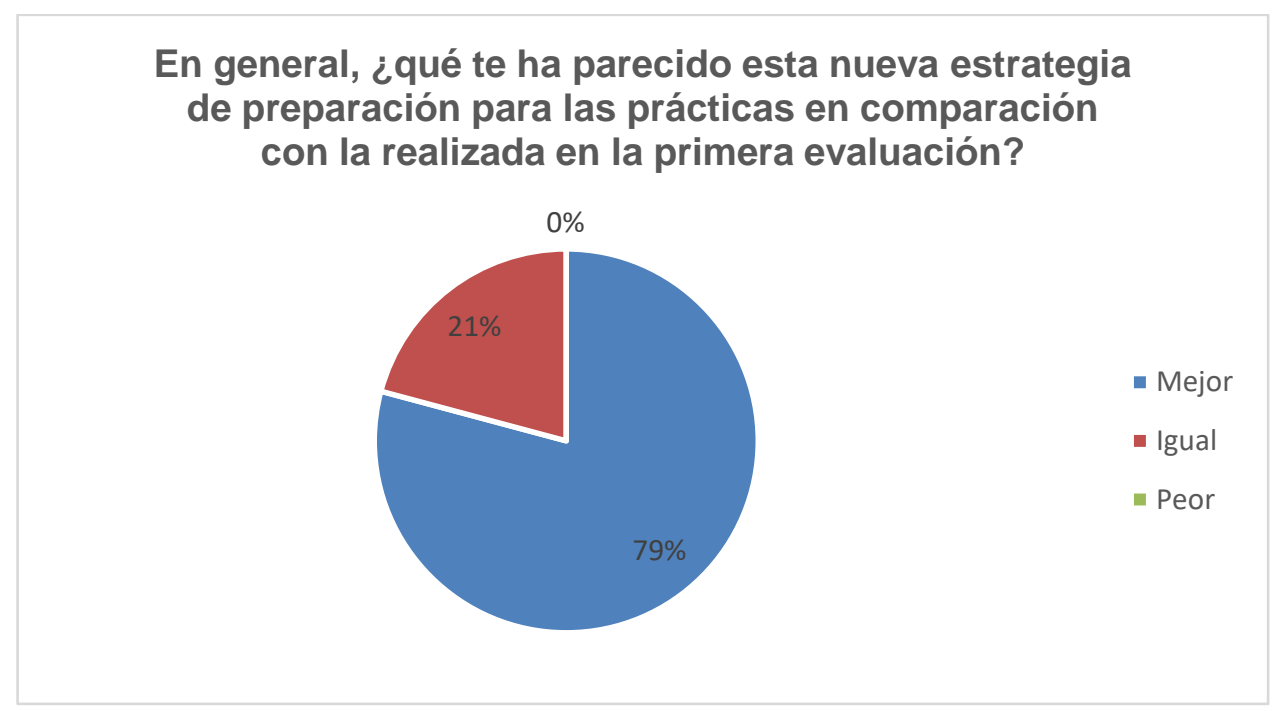

Figura 5. Opinión sobre la propuesta utilizada.

A pesar de que no se ha obtenido una respuesta uniforme o absoluta en esta pregunta, en los datos recogidos de la figura 6 , se aprecia que la opinión general del alumnado es que han adquirido una mejor preparación para realizar las prácticas a través del uso de los recursos propuestos. Estos resultados son más esperanzadores que los que se obtuvieron en el cuestionario previo sobre la preparación para las prácticas externas. 


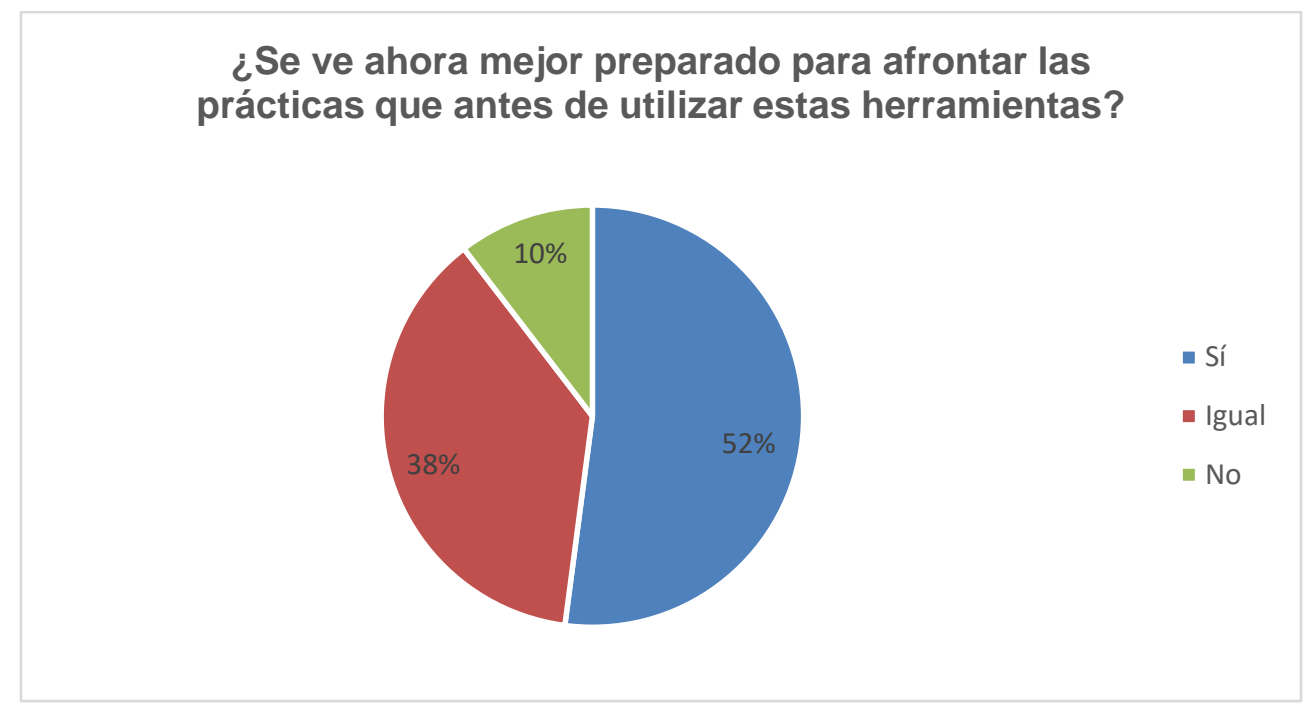

Figura 6. Preparación final para las prácticas externas.

\subsection{Rendimiento académico}

En las figuras 7 y 8 se observa la comparación académica de cada alumno entre la 1. a evaluación y la 2. a evaluación; sin embargo, esto no implica directamente que la variación en las calificaciones se deba únicamente al uso de simuladores virtuales en la materia, pero la incorporación de estos recursos virtuales a la metodología ha sido el principal cambio respecto a los recursos empleados en la 1. a evaluación.

Estas figuras muestran que para la gran mayoría del grupo, salvo algunos casos puntuales, la calificación de la segunda evaluación ha sido igual o superior a la de la 1. a evaluación.

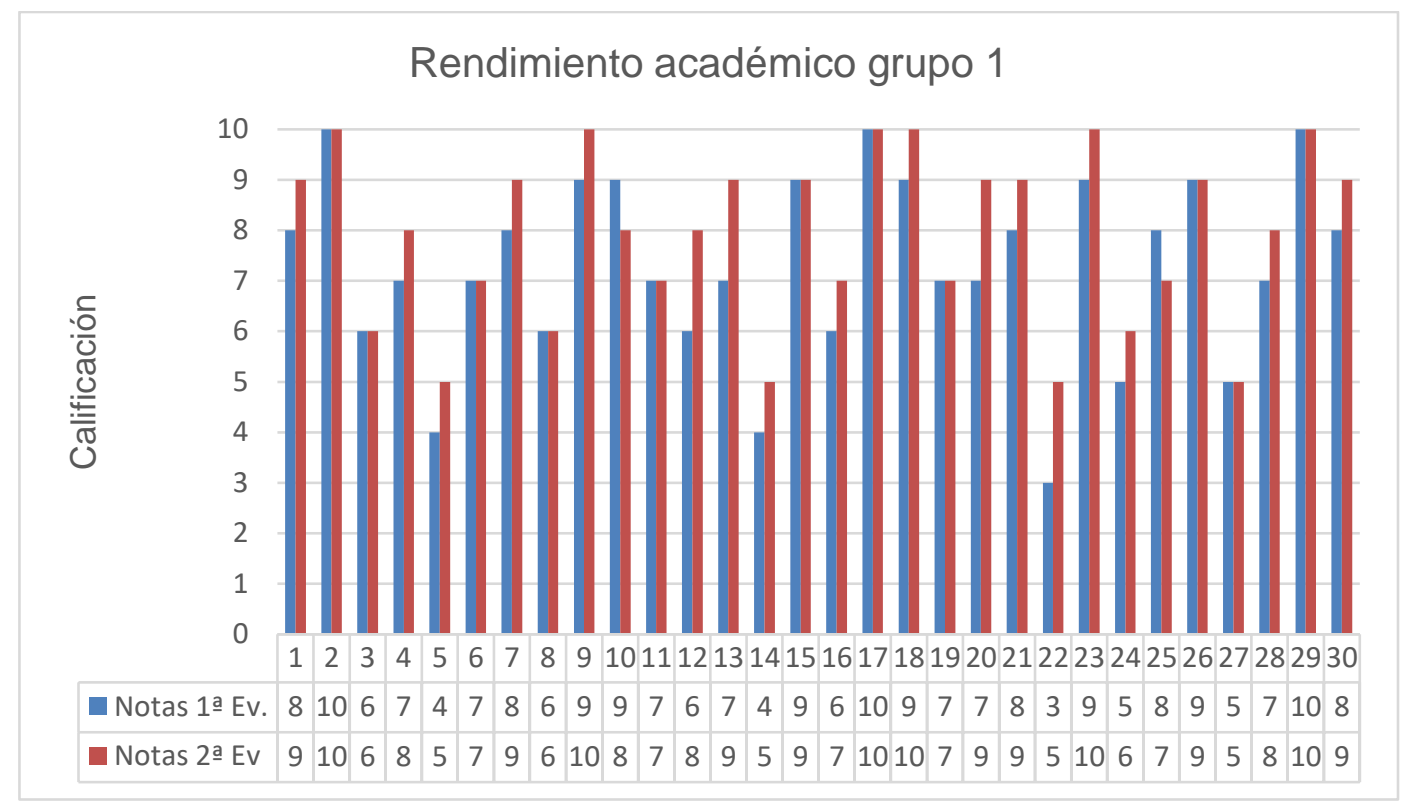

Figura 7. Resultados académicos grupo 1. 


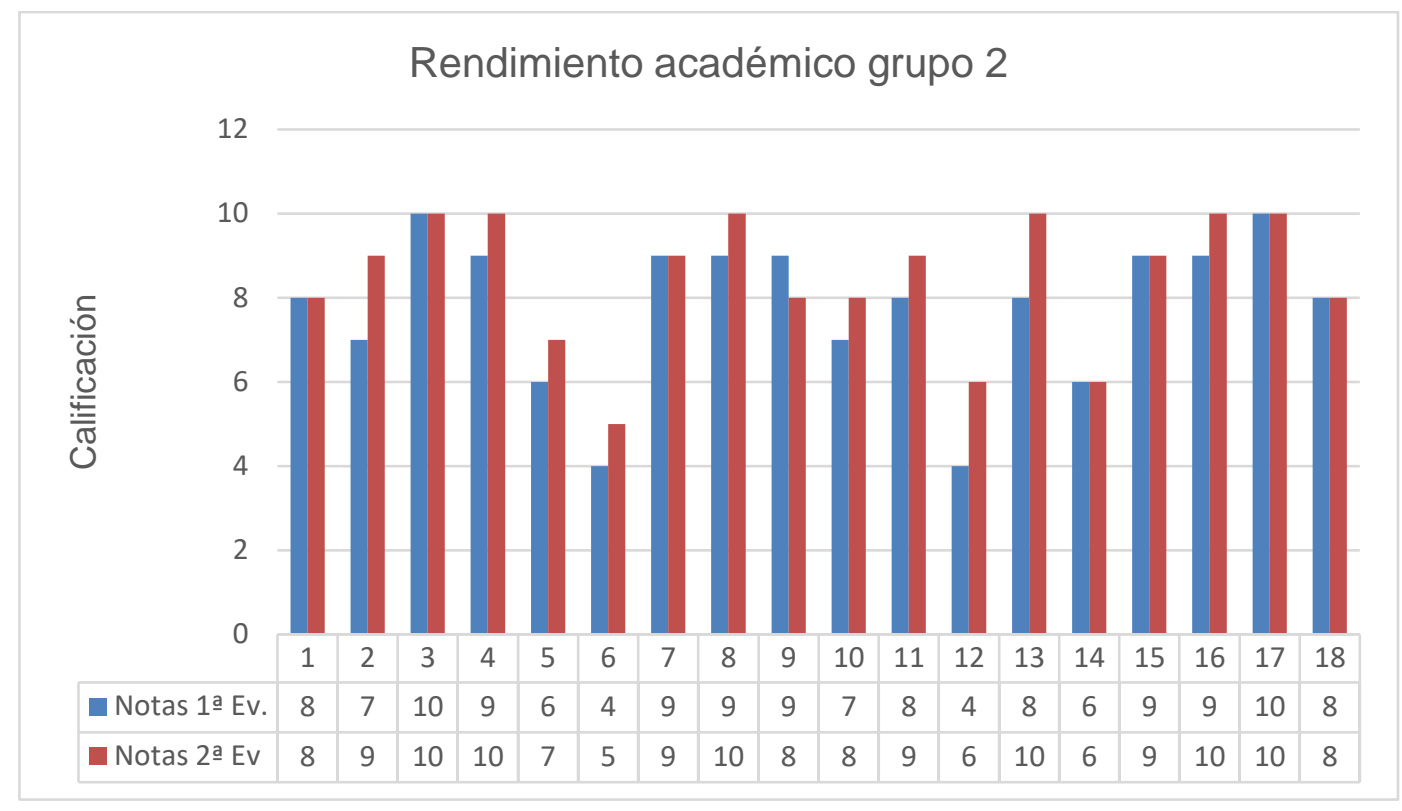

Figura 8. Resultados académicos grupo 2.

Aunque no se puede relacionar directamente con el uso de esta herramienta, se considera que el poder aplicar los conocimientos adquiridos a una situación práctica mediante la simulación, ha ayudado a retener y clarificar los conceptos de estas técnicas, influyendo notablemente en las calificaciones obtenidas.

\subsection{Resultado de la entrevista}

Por último, para finalizar con este apartado de resultados, a continuación se mostrarán algunas respuestas obtenidas en la reunión que se realizó con el tutor de prácticas. Se llevó a cabo una entrevista de tipo semiestructurado, caracterizada por ofrecer una mayor flexibilidad para obtener información.

- Respecto a las expectativas que tenían los alumnos sobre las prácticas y si estas se están cumpliendo:

"En esta fase los alumnos siempre se muestran muy ilusionados por poner en práctica todo lo aprendido durante estos dos años, y más teniendo en cuenta que en el centro no tenemos el aparataje para que puedan practicarlo"

"En las reuniones que realizamos todas las semanas he podido apreciar que vienen muy contentos y el seguimiento que he observado es muy positivo"

- Sobre cómo están realizando las prácticas los alumnos en los centros sanitarios:

"En lo que corresponde a la técnica de Tomografía Computarizada, los alumnos han estado respondiendo muy bien a pesar de tratarse de una de las técnicas más complejas a las que se pueden enfrentar. Aquí en nuestro centro, sí que tenemos aparatos de otras áreas como radiología simple y eso se puede apreciar, los alumnos antes de empezar las prácticas ya han realizado protocolos sobre esto y están muy preparados. Pero por los informes que he podido obtener a estas alturas del módulo, los alumnos también se están desenvolviendo muy bien en el resto de técnicas como TC o Resonancias Magnéticas".

- Su opinión sobre el uso de simuladores virtuales:

"Aunque no conozco ni he trabajado con estos simuladores, si lo veo como una buena opción para una primera toma de contacto. A pesar de que no es lo mismo que trabajar con un paciente, imagino que en el simulador aprenderán lo relacionado con posicionamiento, tratamiento de datos, etc. que les permita hacerse una idea, realmente eso es lo importante, de cómo funciona la TC y los pasos que hay que dar para trabajar con el paciente. Entonces, al no tener el equipo en el centro, esta es una gran alternativa como aproximación a lo que se pueden encontrar en los centros de prácticas". 
- Sobre si ha apreciado alguna diferencia en la preparación para las prácticas de estos alumnos respecto a grupos de cursos anteriores:

"Por los informes que he obtenido, teniendo en cuenta que todavía nos encontramos a mitad del periodo de prácticas, parece que los alumnos, por lo general, están respondiendo muy bien y se han adaptado rápidamente. Estoy muy contenta por la preparación que llevan los alumnos en todas las áreas".

\subsection{Triangulación de datos obtenidos}

El análisis de los datos recogidos, al utilizar más de un instrumento, ofrece la oportunidad de contrastar algunos aspectos y enriquecer las conclusiones.

Se puede observar que los datos obtenidos de los cuestionarios realizados por el alumnado contrastan con la entrevista realizada al tutor. Aunque de forma general los alumnos valoraban positivamente el uso de simuladores virtuales para trabajar la parte práctica, estos datos se obtuvieron antes de que el alumnado comenzara el periodo de Formación en los Centros de Trabajo, mientras que la entrevista se realizó en un momento del curso en el que el tutor ya contaba con algunos informes de cómo se estaban desarrollando dichas prácticas en los centros.

Es por ello que, en la evaluación técnica del instrumento, se ha pretendido destacar la valoración realizada por el docente y el técnico, debido a la experiencia y cercanía de ambos con la realidad práctica del ámbito sanitario que se pretendía trabajar con la simulación.

Con este análisis de los instrumentos utilizados se pretende validar el uso de la herramienta utilizada como alternativa a la preparación práctica del alumnado de Formación Profesional.

\section{DISCUSIÓN Y CONCLUSIONES}

Del análisis de la información obtenida en el apartado anterior, donde se muestran los resultados de usabilidad del simulador y satisfacción de los usuarios evaluados, se obtienen una serie de conclusiones sobre esta investigación, cuyo principal objetivo era diseñar y validar una propuesta metodológica basada en el uso de simuladores virtuales, con la que se pretendía conseguir una mejora en la preparación práctica del alumnado:

- Que el simulador utilizado facilita y permite al alumnado poner en práctica los conocimientos teóricos del módulo.

- Que la herramienta presentada es una gran alternativa a la falta de ambientes prácticos donde los alumnos pueden llevar a cabo distintos protocolos.

- Que el uso de simuladores como complemento a la formación teórica es ampliamente aceptado por el grupo de alumnos con el que se ha llevado a cabo la investigación.

Los resultados obtenidos permiten indicar que se ha alcanzado el objetivo general establecido en la investigación: diseñar y validar una propuesta metodológica basada en el uso de simuladores virtuales, como apoyo a las clases teóricas, y que mejore la preparación práctica del alumnado. Así se resuelve la carencia de experiencias prácticas que acompañen a la teoría (Contreras y Carreño, 2011).

Sin embargo, estos recursos digitales todavía tienen muchas características que mejorar para poder afirmar con rotundidad que pueden llegar a ser un buen instrumento para la formación práctica del alumnado. Como ejemplos de aspectos de estos simuladores valorados por el docente y el técnico de imagen que no alcanzan el nivel de la realidad clínica, se pueden citar que los tiempos de trabajo no son suficientes para asemejarse totalmente a una situación práctica o que, a pesar de ofrecer una información correcta sobre las técnicas empleadas, es necesario actualizar algunas de las técnicas trabajadas mediante estos recursos para añadir los nuevos avances en el ámbito médico. Aun así, el uso de estas herramientas digitales permite la reproducción de situaciones prácticas con suficiente veracidad para conseguir una preparación significativa y realista del alumnado, sobre todo en casos como en el que nos 
enfrentamos en los que por la naturaleza de las técnicas estudiadas y la falta de equipamiento, no hay más alternativas a las explicaciones teóricas para conocer esta práctica.

Con los datos anteriores, se puede afirmar, de forma general, que el uso de simuladores virtuales como estrategia didáctica causa un efecto positivo en el proceso de aprendizaje de los estudiantes (Contreras, García y Ramírez, 2010); y es un método de preparación práctica válido en situaciones donde no se puede trabajar este aspecto con otras técnicas. Además, la adquisición de los contenidos se vuelve más interesante al presentarse en un ámbito distinto a la clase tradicional, hay una mayor participación de los alumnos y se incrementa la motivación en el aula.

De este modo, se demuestra que los simuladores son una estrategia válida en esta rama sanitaria de Formación Profesional y, que permiten conseguir una mejora en la preparación práctica del alumnado, al facilitar que éste se enfrente a situaciones similares a la realidad en un ambiente controlado de trabajo, tal y como exponen Cabrero y Costas (2016).

La simulación virtual puede ser un medio muy eficaz para la adquisición de habilidades y competencias clínicas con las que debe contar un técnico superior de imagen y diagnóstico; si bien, estas herramientas tienen todavía que mejorar para ofrecer a los usuarios una experiencia lo más realista posible.

\section{ENLACES}

Simulador: http://recursostic.educacion.es/fprofesional/simuladores/web/index.php?xml=isanidad-tomografia \&xsl=simulador-idiomas

Cuestionario previo: https://forms.gle/sVoZQ2qA4Ymgism19

Instrumento de evaluación del simulador: https://docs.google.com/document/d/1VMLszr5DcRcykRTtxuV7t4MMYFE6RcBI8RzD567alFE/e dit?usp=sharing

Cuestionario final: https://forms.gle/Li9oHPSspCnKLZLa7

Guion de la entrevista: https://docs.google.com/document/d/16hhmacFoHkOQOn3rMfwL1d0pPJRBSeWR-R-icU DsA/edit?usp=sharing

\section{REFERENCIAS BIBLIOGRÁFICAS}

Alcides, L. (2008). Blended Learning. La nueva formación en Educación Superior. AVANCES Investigación en Ingeniería, 1(9), 95-102. https://bit.ly/39zDKok

Cabero, J. y Costas, J. (2016). La utilización de simuladores para la formación de los alumnos. Prisma Social, 17, 343-372. https://bit.ly/2R3yDXk

Cárdenas, G., Sánchez, B., y Castillo, E. (2016). Desarrollo y evaluación de simuladores virtuales para la enseñanza de competencias en el campo de la salud. Assensus, 1(1), 5973. https://bit.ly/2JuF81e

Contreras, G., García, R. y Ramírez, M. (2010). Uso de simuladores como recurso digital para la transferencia de conocimiento. Apertura, 2(1), 86-100. https://bit.ly/2wTSxgl

Corvetto, M., Bravo, M.P., Montaña, R., Utili, F., Escudero, E., Boza, C., Varas, J. y Dagnino, J. (2013). Simulación en educación médica: una sinopsis. Revista médica de Chile, 141(1), 70-79. https://bit.ly/39wMzPO

Coscollola, M. y Fuentes, M. (2010). Innovación Educativa: Experimentar con las TIC y reflexionar sobre su uso. Pixel-Bit. Revista de Medios y Educación, 36, 171-180. https://bit.ly/2R2f7u7

De Benito, B. y Salinas, J.M. (2016). La investigación basada en diseño en Tecnología Educativa. RIITE. Revista Interuniversitaria de Investigación en Tecnología Educativa, 0 , 44-59. http://dx.doi.org/10.6018/riite/2016/260631 
Franco, I.L. y Álvarez, F.J. (2007). Los simuladores, estrategia formativa en ambientes virtuales de aprendizaje. Revista Virtual Católica del Norte, 21. https://bit.ly/3azWzsP

García, M.R., Reyes, J. y Godínez, G. (2018). Las Tic en la educación superior, innovaciones y retos. RICSH Revista Iberoamericana De Las Ciencias Sociales Y Humanísticas, 6(12), 299 - 316. https://doi.org/10.23913/ricsh.v6i12.135

Gibelli, T. (2014). La investigación basada en diseño para el estudio de una innovación en educación superior que promueve la autorregulación del aprendizaje utilizando TIC. Comunicación presentada en el Congreso Iberoamericano de Ciencia, Tecnología, Innovación y Educación. ISBN: 978-84-7666-210-6 - Artículo 1440. https://bit.ly/2Ux2cTf

González Pérez, A. y De Pablos, J. (2015). Factores que dificultan la integración de las TIC en las aulas. Revista de Investigación Educativa, 33(2), 401-417. http://dx.doi.org/10.6018/rie.33.2.198161

Matia, V. (2016). Formación profesional y formación para el empleo en España: del aprendizaje de tareas al desarrollo de competencias. [Tesis Doctoral, Universidad de Valladolid]. https://bit.ly/39BndjQ

Pin, J.R. y García, P. (2018). Reflexiones sobre la Formación Profesional de Grado Medio y Superior en España. https://bit.ly/2w1J2eD

Rodríguez, K., Maya, M. y Jaén, J. (2012). Educación en Ingenierías: de las clases magistrales a la pedagogía del aprendizaje activo. Ingeniería y Desarrollo, 30(1), 125-142. https://bit.ly/3dLsyby

Salinas, J. (2004). Innovación docente y uso de las TIC en la enseñanza universitaria. Revista Universidad y Sociedad del Conocimiento, 1(1), 1-16. https://bit.ly/3dCSzK6

Silva, J. y Maturana, D. (2017). Una propuesta de modelo para introducir metodologías activas en educación superior. Innovación Educativa, 17(73), 117-131. https://bit.ly/2R1vc3b 


\section{INFORMACIÓN SOBRE LOS AUTORES}

\section{Daniel Romero López}

Universidad de las Islas Baleares

Graduado en Biotecnología en la Universidad de Murcia y en posesión del Máster de Formación del Profesorado; actualmente cursando el Máster Interuniversitario de Tecnología Educativa en la Universidad de las Islas Baleares (UIB). Nivel C1 de inglés a través de la Escuela Oficial de Idiomas. Experiencia docente en el ciclo de Imagen para el diagnóstico y medicina nuclear de Formación Profesional Superior, en los módulos de Tomografía Computarizada y Resonancia Magnética.

Investigación llevada a cabo sobre el uso de simuladores virtuales en el ámbito sanitario de Formación Profesional. Twitter: @dani_romero95

\section{Bárbara de Benito Crosetti}

Universidad de las Islas Baleares

Licenciada en Pedagogía (1991) y doctora en Ciencias de la Educación (2006) por la Universitat de les Illes Balears (UIB).

Miembro del Grupo de Tecnología Educativa (GTE) desde su creación en 1992 hasta la actualidad, donde ha participado en diferentes proyectos de investigación, algunos de ellos de I+D, de diseño de materiales y de análisis, diseño, implementación y evaluación de entornos virtuales de formación.

Líneas actuales de investigación: herramientas y plataformas para la formación en red; videoconferencia en la docencia; diseño de materiales didácticos; estrategias didácticas en entornos virtuales de enseñanza-aprendizaje; sistemas de gestión del conocimiento y el aprendizaje.

Los textos publicados en esta revista están sujetos a una licencia de Reconocimiento 4.0 España de Creative Commons. Puede copiarlos, distribuirlos, comunicarlos públicamente y hacer obras derivadas siempre que reconozca los créditos de las obras (autoría, nombre de la revista, institución editora) de la manera especificada por los autores o por la revista. La licencia completa se puede consultar en:Licencia Creative Commons Atribución-NoComercial-Compartir por igual 4.0 Internacional. 